\title{
Tunable multiplication of the repetition rate of an optical pulse train using dynamic Brillouin gratings in optical fibers
}

\author{
S. Chin ${ }^{1}$, N. Primerov ${ }^{1}$, S. Sales ${ }^{2}$ and L. Thévenaz ${ }^{1}$ \\ 1. Ecole Polytechnique Fédérale de Lausanne, STI-GR-SCI-LT Station 11, CH-1015 Lausanne, Switzerland \\ 2. iTEAM Institute, Universidad Politécnica de Valencia, 46022 Valencia, Spain
}

Recently, all-optical multiplication techniques of the repetition rate of optical pulse trains has been actively searched using a variety of mechanisms, i.e. spectral filtering [1,2], spatial Talbot effects [3,4] and micro-cavity structures [5] since it is believed to be a promising solution for ultrafast transmission in optical communication systems and optical signal processing. We here propose an entirely different approach for realizing the multiplication of the pulse repetition rate with a flexibly tunable multiplication factor. Actually, this technique is based on an all-fiber configuration, which renders the system very attractive for future applications in optical communications. This system makes use of dynamic Brillouin grating (DBG) reflectors implemented in highly birefringent fibers, and the repetition rate of a pulse train is readily reconfigurable by simply changing the number of dynamic grating reflectors positioned in the fiber.

The generation of dynamic Brillouin gratings is based on the process of stimulated Brillouin scattering in polarization maintaining fibers (PMF) [6], and it must be pointed out that the grating appears only at positions along the fiber where two pump pulses are simultaneously present. Figure 1 depicts the schematic diagram for the realization of a pulse repetition rate multiplier. In our experiment, a $60 \mathrm{~cm}-$ long PMF (3 ns transit time) was used as Brillouin gain medium. To generate the dynamic gratings in the fiber, we prepared two Gaussian-shaped pump pulse trains (Pump 1 at optical frequency $v_{1}$ and Pump 2 at $v_{2}$ ) with FWHM duration of $300 \mathrm{ps}$ at a repetition rate of $8 \mathrm{~ns}$. The two pumps were linearly polarized before entering into the PMF along the slow birefringence axis. As a result of the pulses timing, only one dynamic grating was created along the entire PMF through the SBS process. Then a signal pulse train, polarized along the fast axis, with duration of $170 \mathrm{ps}$ at a $8 \mathrm{~ns}$ repetition rate was launched into the PMF. Figure 2(a) shows the time trace of the reflected pulse through the generated single DBG, a simple replica of the original pulse train, analogous to the pulse reflection from a single fiber Bragg grating.

However, by altering the repetition rate of the pump pulses to $4 \mathrm{~ns}$, two gratings were simultaneously created in the PMF, and the time-of-flight interval

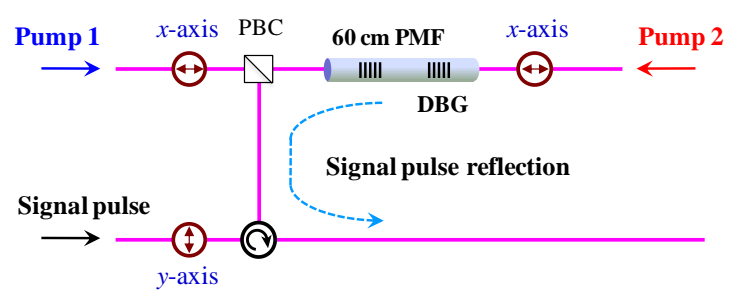

Fig. 1 Schematic diagram to generate multiplication of pulse train repetition rate, based on dynamic grating reflectors in fibers.

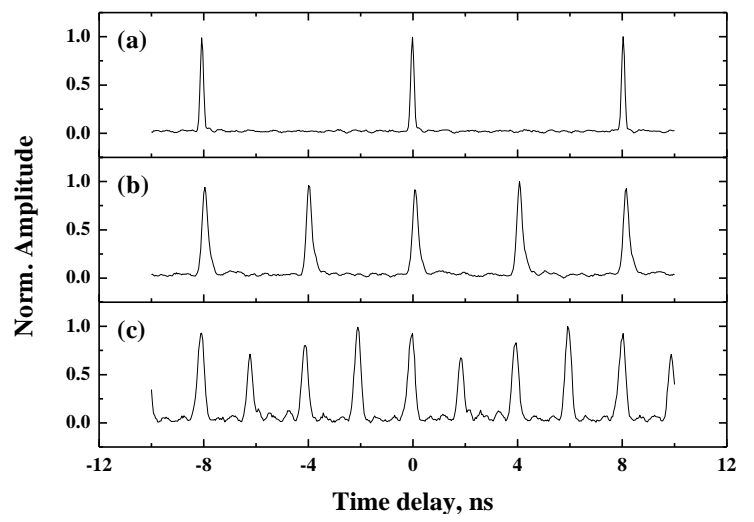

Fig. 2 Time traces of reflected pulse train through: single grating (a), two gratings (b), and four gratings along the fiber.

between the two gratings was measured to be $2 \mathrm{~ns}$ since the 2 pump pulses contrapropagate and their relative velocity is doubled. In this case the repetition rate of the reflected pulse train was doubled, as shown in Figure 2(b). By another doubling of the pumps repetition rate a four-times multiplication was obtained, shown in Figure 2(c). The number of DBG that can be created along the fiber is simply controlled by changing the repetition rate of the pump pulses, which makes the multiplication factor flexibly tunable. The maximum number of grating reflectors, hence the maximum multiplication factor is only limited by the fiber length.

\section{References}

[1] P. Petropoulos, M. Ibsen, M. N. Zervas and D. J. Richardson, "Generation of a $40 \mathrm{GHz}$ pulse stream by pulse multiplication with a sampled fiber Bragg grating, " Opt. Lett. 25, 521-523 (2000).

[2] D. S. Seo, D. E. Leaird, A. M. Weiner, S. Kamei, M. Ishii, A. Sugita and K. Okamoto, "Continuous $500 \mathrm{GHz}$ pulse train generation by repetition rate multiplication using arrayed waveguide grating," Electron. Lett. 39, 1138-1140 (2003).

[3] J. Azana and M. A. Muriel, "Temporal Talbot effect in fiber gratings and its applications," Appl. Opt. 38, 6700 (1999).

[4] J. Caraquitena, Z, Jiang, D. E. Leaird and A. M. Weiner, "Tunable pulse repetition rate multiplication using phase-only line-by-line pulse shaping," Opt. Lett. 32, 716-718 (2007).

[5] M. A. Preciado and M. A. Muriel, "All-pass optical structures for repetition rate multiplication,” Opt. Express 16, 11162-11168 (2008) [6] K. Y. Song, W. Zou, Z. He and K. Hotate, "All-optical dynamic grating generation based on Brillouin scattering in polarization maintaining fiber," Opt. Lett. 33, 926-928 (2008). 